\title{
The world according to zebrafish: how neural circuits generate behavior
}

\author{
Germán Sumbre ${ }^{1,2,3 *}$ and Gonzalo G. de Polavieja ${ }^{4,5 *}$ \\ 1 Ecole Normale Supérieure, Institut de Biologie de I'ENS, Paris, France \\ 2 Inserm, U1024, Paris, France \\ ${ }^{3}$ CNRS, UMR 8197, Paris, France \\ ${ }^{4}$ Instituto Cajal, Consejo Superior de Investigaciones Científicas, Madrid, Spain \\ ${ }^{5}$ Champalimaud Neuroscience Programme, Champalimaud Center for the Unknown, Lisbon, Portugal \\ *Correspondence: sumbre@biologie.ens.fr; gonzalo.polavieja@neuro.fchampalimaud.org
}

Edited and reviewed by:

Florian Engert, Harvard University, USA

Keywords: zebrafish, behavior, neuronal circuit dynamics, neuroanatomy, Models of human brain disorders

Understanding how the brain controls motor behavior and generates cognitive functions still remains one of the most challenging goals in science and neuroscience in particular.

Toward this goal it is important to use a multidisciplinary approach involving genetics, molecular biology, optics, ethology, neurobiology, and mathematical modeling. This strategy is most efficient when using animal models with relatively simple nervous systems still capable of producing complex motor behaviors. Genetically tractable models enable labeling specific neurons and monitoring and manipulating neuronal activity of single cells or entire circuits via optogenetics (Fenno et al., 2011; Akerboom et al., 2013; Aston-Jones and Deisseroth, 2013; Chen et al., 2013; Marvin et al., 2013).

The zebrafish Danio rerio is a small shoaling tropical freshwater fish native to rivers of south Asia. It is a member of the teleostei infraclass, a monophyletic group that emerged $\sim 340$ million years ago (Amores et al., 2011). Compared to other vertebrate species, teleost fish underwent an additional round of whole-genome duplication (Meyer and Schartl, 1999).

Zebrafish has been used for developmental and genetic studies since the late 1950s. By the 1980s, zebrafish was already used as a genetically tractable organism. In 2001 the zebrafish genomesequencing project was launched and recently its protein-coding genes were compared to those of humans (Friedrich et al., 2010; Howe et al., 2013). This large-scale project showed that zebrafish have 26,206 protein-coding genes (Collins et al., 2012), with $\sim 70 \%$ of human genes having at least one obvious zebrafish ortholog (Howe et al., 2013).

The combination of high-throughput mutagenesis and TILLING (Wienholds et al., 2003) or specifically targeted DNA sequence mutations [Zinc-finger nucleases (Doyon et al., 2008), TALENS (Sander et al., 2011) and CRISPR (Hwang et al., 2013)], enable DNA precise editing and thus the generation of transgenic and/or specific mutant zebrafish lines. Among this increasing collection of available mutants, several were identified as vertebrate models of certain human neurodevelopmental, neurological, and neurodegenerative syndromes and diseases [e.g., Parkinson's (Lam et al., 2005; Flinn et al., 2008), Alzheimer's (Newman et al., 2007, 2011), Rett's syndrome (Pietri et al.,
2013), ALS (Gibbs et al., 1976; Burrill and Easter, 1994; Da Costa et al., 2014), tinnitus (Wu et al., 2014), psychiatric disorders (Norton, 2013), Huntington's disease (Schiffer et al., 2007), Lowe's syndrome (Ramirez et al., 2012), and more (Sager et al., 2010)].

Furthermore, large-scale enhancer-trap screens in combination with DNA insertion methods (e.g., Tol2, Kawakami and Shima, 1999), bioinformatics and the Gal4/UAS system generated a vast collection of transgenic fish and a large database of tissue/cell-type specific promoters (Scott et al., 2007; Asakawa et al., 2008).

An additional advantage of the zebrafish larva model is its transparent skin, small size and the fact that it mainly uses cutaneous breathing (up to $\sim 14$ days post-fertilization, dpf). These three characteristics make possible to restrain larvae in a drop of low-melting agarose without the use of any paralyzers or anesthetics, in intact conditions, without the use of surgical procedures to expose and image the brain.

With the development of recent state-of-the-art optical techniques including two-photon scanning microscopy (Ahrens et al., 2012; Portugues et al., 2014), Single plain illumination microscopy (Ahrens et al., 2013b; Panier et al., 2013), lightfield microscopy (Broxton et al., 2013), and Spatial light modulator microscopy (Quirin et al., 2013), the entire brain can be now simultaneously imaged and its activity monitored with single or near single-cell resolution. On the other hand, fiber optics (Miri et al., 2011; Kubo et al., 2014), Digital micromirror devices (Wyart et al., 2009), and holographic pattern illumination (Vaziri and Emiliani, 2012) can be used to stimulate optogenetic tools in single cells or large neuronal circuits.

The combination of all these techniques together with the larva's small size and skin transparency enable monitoring in toto brain dynamics and manipulate its activity in an intact, non-anesthetized, non-paralyzed vertebrate (Ahrens et al., 2013b; Panier et al., 2013; Portugues et al., 2014).

From a behavioral point of view, upon hatching the larva needs to immediately catch prey and avoid predators in order to survive. This strong evolutionary pressure leads to a rapid development of functional sensory systems in general, and vision in particular, 
creating a reach repertoire of visuo-motor behaviors (Fleisch and Neuhauss, 2006; Portugues and Engert, 2009). For example, the startle escape response [a flash of light induces a directional swimming behavior (Burgess and Granato, 2007)], optokinetic response [OKR, compensatory eye saccades evoked by coherent field motion (Rinner et al., 2005; Mueller and Neuhauss, 2010)], optomotor response [OMR, compensatory tail movements evoked by coherent field motion (Orger et al., 2000)], dorsal-light response (DLR, Neuhauss, 2003), feeding behavior (Budick and O'Malley, 2000), and eye lateralization [preferential use of one eye over the other depending on the type of visual stimulus (Miklósi and Andrew, 2006)]. Furthermore, they also show rheotaxis (Olszewski et al., 2012), odor and gustatory-induced behaviors (Mathuru et al., 2012; Boyer et al., 2013), learning and memory (Aizenberg and Schuman, 2011; Valente et al., 2012; Roberts et al., 2013), and circadian rhythms and sleep (Naumann et al., 2010; Chiu and Prober, 2013; Elbaz et al., 2013), among others.

At the juvenile and adult stages, zebrafish develop more complex behaviors such as social learning, shoaling, group decision making and learning, courtship, territoriality, and hierarchy (Arganda et al., 2012; Oliveira, 2013).

With all these multidisciplinary advantages in hand and a relative simple nervous system $[\sim 100,000$ neurons at $7 \mathrm{dpf}$ (Hill et al., 2003; Naumann et al., 2010)], still with a well-conserved vertebrate structure, the zebrafish is becoming an emerging experimental model in neurosciences and neuroethology (Friedrich et al., 2010).

Recent studies have shown that it is also possible to monitor and/or manipulate neuronal dynamics in partially agaroserestrained behaving intact larvae and therefore correlate neuronal activity and motor behavior. Moreover, close-loop visual virtual reality can be used so larvae can get visual feedback of their own acts despite being immobilized (Ahrens et al., 2012, 2013a).

Alternatively, although lacking cellular resolution, using transgenic larvae expressing a bioluminescence protein such as GFPAequorin expressed in specific cell populations, it is possible to monitor brain activities in unrestrained freely behaving animals (Naumann et al., 2010).

Furthermore, due to the zebrafish ex-uterus development all embryonic and larval developmental stages following fecundation are accessible for imaging.

The combination of the genetic and optical state-of-the-art techniques with the zebrafish experimental model is yielding high-dimensional large data sets pushing the limits of current data analysis standards, forcing for the development of new methodologies and novel theoretical models.

A future challenge in the field will be monitoring whole-brain activity with near single-neuron resolution from multiple freely behaving and socially interacting individuals.

In this topic we have gathered a collection of original articles, reviews, and opinions covering a wide-spectrum of topics from behavior up to whole-brain activity recordings, both in wild type and in neurological human-syndrome models, providing an overview of current state and future directions of zebrafish circuits neuroscience and behavior research field.
We have organized this eBook in 4 different chapters:

\section{Neuroanatomy}

2. Neuronal circuit dynamics

3. Behavior

4. Models of brain disorders and addiction

The zebrafish model in combination with recently developed imaging techniques, optogenetics, and sophisticated mathematical methods for analysis of the acquired large data sets is bringing us closer than ever before to the understanding of how brain dynamics relates to behavior.

\section{REFERENCES}

Ahrens, M. B., Huang, K. H., Narayan, S., Mensh, B. D., and Engert, F. (2013a). Two-photon calcium imaging during fictive navigation in virtual environments. Front. Neural Circuits 7:104. doi: 10.3389/fncir.2013. 00104

Ahrens, M. B., Li, J. M., Orger, M. B., Robson, D. N., Schier, A. F., Engert, F., et al. (2012). Brain-wide neuronal dynamics during motor adaptation in zebrafish. Nature 485, 471-477. doi: 10.1038/nature11057

Ahrens, M. B., Orger, M. B., Robson, D. N., Li, J. M., and Keller, P. J. (2013b). Whole-brain functional imaging at cellular resolution using light-sheet microscopy. Nat. Methods 10, 413-420. doi: 10.1038/ nmeth. 2434

Aizenberg, M., and Schuman, E. M. (2011). Cerebellar-dependent learning in larval zebrafish. J. Neurosci. 31, 8708-8712. doi: 10.1523/JNEUROSCI.656510.2011

Akerboom, J., Carreras Calderón, N., Tian, L., Wabnig, S., Prigge, M., Tolö, J., et al. (2013). Genetically encoded calcium indicators for multi-color neural activity imaging and combination with optogenetics. Front. Mol. Neurosci. 6:2. doi: 10.3389/fnmol.2013.00002

Amores, A., Catchen, J., Ferrara, A., Fontenot, Q., and Postlethwait, J. H. (2011). Genome evolution and meiotic maps by massively parallel DNA sequencing: spotted gar, an outgroup for the teleost genome duplication. Genetics 188, 799-808. doi: 10.1534/genetics.111.127324

Arganda, S., Pérez-Escudero, A., and de Polavieja, G. G. (2012). A common rule for decision making in animal collectives across species. Proc. Natl. Acad. Sci. U.S.A. 109, 20508-20513. doi: 10.1073/pnas.1210664109

Asakawa, K., Suster, M. L., Mizusawa, K., Nagayoshi, S., Kotani, T., Urasaki, A., et al. (2008). Genetic dissection of neural circuits by Tol 2 transposon-mediated Gal4 gene and enhancer trapping in zebrafish. Proc. Natl. Acad. Sci. U.S.A. 105, 1255-1260. doi: 10.1073/pnas.0704963105

Aston-Jones, G., and Deisseroth, K. (2013). Recent advances in optogenetics and pharmacogenetics. Brain Res. 1511, 1-5. doi: 10.1016/j.brainres.2013. 01.026

Boyer, B., Ernest, S., and Rosa, F. (2013). Egr-1 induction provides a genetic response to food aversion in zebrafish. Front. Behav. Neurosci. 7:51. doi: 10.3389/fnbeh.2013.00051

Broxton, M., Grosenick, L., Yang, S., Cohen, N., Andalman, A., Deisseroth, K., et al. (2013). Wave optics theory and 3-D deconvolution for the light field microscope. Opt. Express 21, 25418-25439. doi: 10.1364/OE.21. 025418

Budick, S. A., and O’Malley, D. M. (2000). Locomotor repertoire of the larval zebrafish: swimming, turning and prey capture. J. Exp. Biol. 203, 2565-2579.

Burgess, H. A., and Granato, M. (2007). Modulation of locomotor activity in larval zebrafish during light adaptation. J. Exp. Biol. 210, 2526-2539. doi: 10.1242/jeb.003939

Burrill, J. D., and Easter, S. S. (1994). Development of the retinofugal projections in the embryonic and larval zebrafish (Brachydanio rerio). J. Comp. Neurol. 346, 583-600. doi: 10.1002/cne.903460410

Chen, T.-W., Wardill, T. J., Sun, Y., Pulver, S. R., Renninger, S. L., Baohan, A., et al. (2013). Ultrasensitive fluorescent proteins for imaging neuronal activity. Nature 499, 295-300. doi: 10.1038/nature12354 
Chiu, C. N., and Prober, D. A. (2013). Regulation of zebrafish sleep and arousal states: current and prospective approaches. Front. Neural Circuits 7:58. doi: 10.3389/fncir.2013.00058

Collins, J. E., White, S., Searle, S. M. J., and Stemple, D. L. (2012). Incorporating RNA-seq data into the zebrafish Ensembl genebuild. Genome Res. 22, 2067-2078. doi: 10.1101/gr.137901.112

Da Costa, M. M. J., Allen, C. E., Higginbottom, A., Ramesh, T., Shaw, P. J., and McDermott, C. J. (2014). A new zebrafish model produced by TILLING of SOD1-related amyotrophic lateral sclerosis replicates key features of the disease and represents a tool for in vivo therapeutic screening. Dis. Model. Mech. 7, 73-81. doi: 10.1242/dmm.012013

Doyon, Y., McCammon, J. M., Miller, J. C., Faraji, F., Ngo, C., Katibah, G. E., et al. (2008). Heritable targeted gene disruption in zebrafish using designed zinc-finger nucleases. Nat. Biotechnol. 26, 702-708. doi: 10.1038/nbt1409

Elbaz, I., Foulkes, N. S., Gothilf, Y., and Appelbaum, L. (2013). Circadian clocks, rhythmic synaptic plasticity and the sleep-wake cycle in zebrafish. Front. Neural Circuits 7:9. doi: 10.3389/fncir.2013.00009

Fenno, L., Yizhar, O., and Deisseroth, K. (2011). The development and application of optogenetics. Annu. Rev. Neurosci. 34, 389-412. doi: 10.1146/annurev-neuro061010-113817

Fleisch, V. C., and Neuhauss, S. C. F. (2006). Visual behavior in zebrafish. Zebrafish 3, 191-201. doi: 10.1089/zeb.2006.3.191

Flinn, L., Bretaud, S., Lo, C., Ingham, P. W., and Bandmann, O. (2008). Zebrafish as a new animal model for movement disorders. J. Neurochem. 106, 1991-1997. doi: 10.1111/j.1471-4159.2008.05463.x

Friedrich, R. W., Jacobson, G. A., and Zhu, P. (2010). Circuit neuroscience in zebrafish. Curr. Biol. 20, R371-R381. doi: 10.1016/j.cub.2010. 02.039

Gibbs, H., McCall, S., and Venkatesan, T. (1976). Differential gain and bistability using a sodium-filled fabry-perot interferometer. Phys. Rev. Lett. 36, 1135-1138. doi: 10.1103/PhysRevLett.36.1135

Hill, A., Howard, C. V., Strahle, U., and Cossins, A. (2003). Neurodevelopmental defects in zebrafish (Danio rerio) at environmentally relevant dioxin (TCDD) concentrations. Toxicol. Sci. 76, 392-399. doi: 10.1093/toxsci/ kfg241

Howe, K., Clark, M. D., Torroja, C. F., Torrance, J., Berthelot, C., Muffato, M., et al. (2013). The zebrafish reference genome sequence and its relationship to the human genome. Nature 496, 498-503. doi: 10.1038/nature12111

Hwang, W. Y., Fu, Y., Reyon, D., Maeder, M. L., Tsai, S. Q., Sander, J. D., et al. (2013). Efficient genome editing in zebrafish using a CRISPR-Cas system. Nat. Biotechnol. 31, 227-229. doi: 10.1038/nbt.2501

Kawakami, K., and Shima, A. (1999). Identification of the Tol 2 transposase of the medaka fish Oryzias latipes that catalyzes excision of a nonautonomous Tol2 element in zebrafish Danio rerio. Gene 240, 239-244.

Kubo, F., Hablitzel, B., Dal Maschio, M., Driever, W., Baier, H., and Arrenberg, A. B. (2014). Functional architecture of an optic flow-responsive area that drives horizontal eye movements in zebrafish. Neuron 81, 1344-1359. doi: 10.1016/j.neuron.2014.02.043

Lam, C. S., Korzh, V., and Strahle, U. (2005). Zebrafish embryos are susceptible to the dopaminergic neurotoxin MPTP. Eur. J. Neurosci. 21, 1758-1762. doi: 10.1111/j.1460-9568.2005.03988.x

Marvin, J. S., Borghuis, B. G., Tian, L., Cichon, J., Harnett, M. T., Akerboom, J., et al. (2013). An optimized fluorescent probe for visualizing glutamate neurotransmission. Nat. Methods 10, 162-170. doi: 10.1038/ nmeth.2333

Mathuru, A. S., Kibat, C., Cheong, W. F., Shui, G., Wenk, M. R., Friedrich, R. W., et al. (2012). Chondroitin fragments are odorants that trigger fear behavior in fish. Curr. Biol. 22, 538-544. doi: 10.1016/j.cub.2012.01.061

Meyer, A., and Schartl, M. (1999). Gene and genome duplications in vertebrates: the one-to-four (-to-eight in fish) rule and the evolution of novel gene functions. Curr. Opin. Cell Biol. 11, 699-704.

Miklósi, A., and Andrew, R. J. (2006). The zebrafish as a model for behavioral studies. Zebrafish 3, 227-234. doi: 10.1089/zeb.2006.3.227

Miri, A., Daie, K., Arrenberg, A. B., Baier, H., Aksay, E., and Tank, D. W. (2011). Spatial gradients and multidimensional dynamics in a neural integrator circuit. Nat. Neurosci. 14, 1150-1159. doi: 10.1038/nn.2888

Mueller, K. P., and Neuhauss, S. C. F. (2010). Quantitative measurements of the optokinetic response in adult fish. J. Neurosci. Methods 186, 29-34. doi: 10.1016/j.jneumeth.2009.10.020
Naumann, E. A., Kampff, A. R., Prober, D. A., Schier, A. F., and Engert, F. (2010). Monitoring neural activity with bioluminescence during natural behavior. Nat. Neurosci. 13, 513-520. doi: 10.1038/nn.2518

Neuhauss, S. C. F. (2003). Behavioral genetic approaches to visual system development and function in zebrafish. J. Neurobiol. 54, 148-160. doi: 10.1002/neu. 10165

Newman, M., Musgrave, I. F., Musgrave, F. I., and Lardelli, M. (2007). Alzheimer disease: amyloidogenesis, the presenilins and animal models. Biochim. Biophys. Acta 1772, 285-297. doi: 10.1016/j.bbadis.2006.12.001

Newman, M., Verdile, G., Martins, R. N., and Lardelli, M. (2011). Zebrafish as a tool in Alzheimer's disease research. Biochim. Biophys. Acta 1812, 346-352. doi: 10.1016/j.bbadis.2010.09.012

Norton, W. H. J. (2013). Toward developmental models of psychiatric disorders in zebrafish. Front. Neural Circuits 7:79. doi: 10.3389/fncir.2013.00079

Oliveira, R. F. (2013). Mind the fish: zebrafish as a model in cognitive social neuroscience. Front. Neural Circuits 7:131. doi: 10.3389/fncir.2013.00131

Olszewski, J., Haehnel, M., Taguchi, M., and Liao, J. C. (2012). Zebrafish larvae exhibit rheotaxis and can escape a continuous suction source using their lateral line. PLoS ONE 7:e36661. doi: 10.1371/journal.pone.0036661

Orger, M. B., Smear, M. C., Anstis, S. M., and Baier, H. (2000). Perception of Fourier and non-Fourier motion by larval zebrafish. Nat. Neurosci. 3, 1128-1133. doi: $10.1038 / 80649$

Panier, T., Romano, S. A., Olive, R., Pietri, T., Sumbre, G., Candelier, R., et al. (2013). Fast functional imaging of multiple brain regions in intact zebrafish larvae using selective plane illumination microscopy. Front. Neural Circuits 7:65. doi: $10.3389 /$ fncir.2013.00065

Pietri, T., Roman, A.-C., Guyon, N., Romano, S. A., Washbourne, P., Moens, C. B., et al. (2013). The first mecp2-null zebrafish model shows altered motor behaviors. Front. Neural Circuits 7:118. doi: 10.3389/fncir.2013.00118

Portugues, R., and Engert, F. (2009). The neural basis of visual behaviors in the larval zebrafish. Curr. Opin. Neurobiol. 19, 644-647. doi: 10.1016/j.conb.2009.10.007

Portugues, R., Feierstein, C. E., Engert, F., and Orger, M. B. (2014). Whole-brain activity maps reveal stereotyped, distributed networks for visuomotor behavior. Neuron 81, 1328-1343. doi: 10.1016/j.neuron.2014.01.019

Quirin, S., Peterka, D. S., and Yuste, R. (2013). Instantaneous three-dimensional sensing using spatial light modulator illumination with extended depth of field imaging. Opt. Express 21, 16007-16021. doi : 10.1364/OE.21.016007

Ramirez, I. B.-R., Pietka, G., Jones, D. R., Divecha, N., Alia, A., Baraban, S. C., et al. (2012). Impaired neural development in a zebrafish model for Lowe syndrome. Hum. Mol. Genet. 21, 1744-1759. doi: 10.1093/hmg/ddr608

Rinner, O., Rick, J. M., and Neuhauss, S. C. F. (2005). Contrast sensitivity, spatial and temporal tuning of the larval zebrafish optokinetic response. Invest Ophthalmol. Vis. Sci. 46, 137-142. doi: 10.1167/iovs.04-0682

Roberts, A. C., Bill, B. R., and Glanzman, D. L. (2013). Learning and memory in zebrafish larvae. Front. Neural Circuits 7:126. doi: 10.3389/fncir. 2013.00126

Sager, J. J., Bai, Q., and Burton, E. A. (2010). Transgenic zebrafish models of neurodegenerative diseases. Brain Struct. Funct. 214, 285-302. doi: 10.1007/s00429009-0237-1

Sander, J. D., Cade, L., Khayter, C., Reyon, D., Peterson, R. T., Joung, J. K., et al. (2011). Targeted gene disruption in somatic zebrafish cells using engineered TALENs. Nat. Biotechnol. 29, 697-698. doi: 10.1038/nbt.1934

Schiffer, N. W., Broadley, S. A., Hirschberger, T., Tavan, P., Kretzschmar, H. A., Giese, A., et al. (2007). Identification of anti-prion compounds as efficient inhibitors of polyglutamine protein aggregation in a zebrafish model. J. Biol. Chem. 282, 9195-9203. doi: 10.1074/jbc.M607865200

Scott, E. K., Mason, L., Arrenberg, A. B., Ziv, L., Gosse, N. J., Xiao, T., et al. (2007) Targeting neural circuitry in zebrafish using GAL4 enhancer trapping. Nat. Methods 4, 323-326. doi: 10.1038/nmeth1033

Valente, A., Huang, K.-H., Portugues, R., and Engert, F. (2012). Ontogeny of classical and operant learning behaviors in zebrafish. Learn. Mem. 19, 170-177. doi: 10.1101/lm.025668.112

Vaziri, A., and Emiliani, V. (2012). Reshaping the optical dimension in optogenetics. Curr. Opin. Neurobiol. 22, 128-137. doi: 10.1016/j.conb.2011. 11.011

Wienholds, E., van Eeden, F., Kosters, M., Mudde, J., Plasterk, R. H. A., and Cuppen, E. (2003). Efficient target-selected mutagenesis in zebrafish. Genome Res. 13, 2700-2707. doi: 10.1101/gr.1725103 
Wu, C., Sharma, K., Laster, K., Hersi, M., Torres, C., Lukas, T. J., et al. (2014). Kcnq1-5 (Kv7.1-5) potassium channel expression in the adult zebrafish. BMC Physiol. 14:1. doi: 10.1186/1472-6793-14-1

Wyart, C., Del Bene, F., Warp, E., Scott, E. K., Trauner, D., Baier, H., et al. (2009). Optogenetic dissection of a behavioural module in the vertebrate spinal cord. Nature 461, 407-410. doi: 10.1038/nature 08323

Conflict of Interest Statement: The authors declare that the research was conducted in the absence of any commercial or financial relationships that could be construed as a potential conflict of interest.
Received: 07 April 2014; accepted: 11 July 2014; published online: 30 July 2014.

Citation: Sumbre G and de Polavieja GG (2014) The world according to zebrafish: how neural circuits generate behavior. Front. Neural Circuits 8:91. doi: 10.3389/fncir. 2014.00091

This article was submitted to the journal Frontiers in Neural Circuits.

Copyright (C) 2014 Sumbre and de Polavieja. This is an open-access article distributed under the terms of the Creative Commons Attribution License (CC BY). The use, distribution or reproduction in other forums is permitted, provided the original author(s) or licensor are credited and that the original publication in this journal is cited, in accordance with accepted academic practice. No use, distribution or reproduction is permitted which does not comply with these terms. 